\title{
Ability of Laboratory Findings upon Admission to Predict Lung Involvement and Its Severity in COVID-19 Patients Requiring Hospitalization
}

\author{
Yusuf Ata1, ${ }^{*}$, Ahmet Kagan As ${ }^{1}$, Mesut Engin ${ }^{1}$, Nurcan Kacmaz Kat ${ }^{2}$, Tirdad Setayeshi², Sadik Ahmet Sunbul ${ }^{1}$, \\ Cuneyt Eris ${ }^{1}$, Ufuk Aydin ${ }^{1}$, Filiz Ata ${ }^{3}$ and Tamer Turk ${ }^{1}$
}

${ }^{1}$ University of Health Sciences, Bursa Yuksek Ihtisas Training and Research Hospital, Department of Cardiovascular Surgery, Bursa, Turkey

2University of Health Sciences, Bursa Yuksek Ihtisas Training and Research Hospital, Department of Radiology, Bursa, Turkey

${ }^{3}$ University of Health Sciences, Bursa Yuksek Ihtisas Training and Research Hospital, Department of Anesthesiology and Reanimation, Bursa, Turkey

* Corresponding author: Yusuf Ata, University of HealthSciences, Bursa Yuksek Ihtisas Training and Research Hospital, Department of Cardiovascular Surgery, Mimar Sinan Town, Emniyet Street, Yıldırım/BURSA, Turkey. Tel: +902242955000; Email: yasefata@hotmail.com

Received 2021 May 31; Revised 2021 July 08; Accepted 2021 October 14.

\begin{abstract}
Background: The struggle of humanity with Coronavirus disease 2019 (COVID-19) infection, which affected the whole world and caused severe social and health crises, continues without deceleration.

Objectives: This study aimed to determine the relationship between the abnormal laboratory parameters upon admission and the intensity of lung involvement using chest computerized tomography severity score (CT-SS).

Methods: This single-center study evaluated a total of 242 patients who were admitted to our hospital due to COVID-19 with positive polymerase chain reaction (PCR) test results. The patients were divided into three groups of no involvement on thorax CT images, mild involvement, and moderate-severe involvement.

Results: The mean ages of groups $1(n=42), 2(n=123)$, and $3(n=77)$ were $38 \pm 10.6,56.3 \pm 16$, and $61 \pm 15.6$ years, respectively $(P<0.001)$. The three groups showed significant differences in terms of hypertension, diabetes mellitus, heart rate, oxygen saturation, lymphocyte count, platelet-lymphocyte ratio (PLR), systemic immune inflammation index (SII), ferritin, troponin-I, erythrocyte sedimentation rate (ESR), and c-reactive protein (CRP) values $(\mathrm{P}<0.001)$. The CRP $(\mathrm{R}=0.545, \mathrm{P}<0.001)$, ferritin $(\mathrm{R}=0.481, \mathrm{P}<0.001)$, and SII $(\mathrm{R}=0.473$, $\mathrm{P}<0.001)$ were moderately and positively correlated with CT-SS, while neutrophil-lymphocyte ratio $(\mathrm{R}=0.404, \mathrm{P}<0.001), \mathrm{PLR}(\mathrm{R}=0.371$, $\mathrm{P}<0.001)$, and ESR $(\mathrm{R}=0.327, \mathrm{P}<0.001)$ were mildly and positively correlated with CT-SS.

Conclusion: The results of the present study showed that elevation in CRP, as well as ferritin and SII values upon admission to the hospital was significantly correlated with CT-SS. The results also revealed that the presence of lung parenchyma involvement might be predicted in PCR positive COVID-19 patients without the need for thorax CT. Furthermore, it is believed that this information will provide great convenience to the clinicians who first welcome the patient in terms of predicting COVID-19 lung involvement.
\end{abstract}

Keywords: Computed tomography, COVID-19, Inflammation, Pandemic

\section{Background}

Coronavirus disease 2019 (COVID-19) went down in history as a disease that first appeared in Wuhan province, China, in December 2019, caused an explosive spread within a very short time, and created a worldwide health crisis. The disease is of zoonotic origin and occurs due to the newly described virus named severe acute respiratory syndrome coronavirus 2 (SARS-CoV-2), a member of the coronavirus family with seven different species known to infect humans to date (1).

COVID-19 is predominantly a disease with fever and cough complaints. Although its symptomatology is highly variable, it exhibits a severe clinical picture in approximately $20 \%$ of patients (2). In this severe group, the mortality rate is around 3\% (3). Changes in some laboratory parameters, especially those with prognostic value are remarkable. Laboratory parameters, such as d-dimer, troponin-I, lymphocyte amount, c-reactive protein (CRP) value, and lactate dehydrogenase (LDH) show disease-specific changes (4). However, the clinical form with pneumonia and lung involvement is also important. There are typical and atypical findings for the diagnosis of COVID-19 detected by computed tomography (CT). Typical findings are bilateral ground-glass appearance, peripheral and dorsal consolidation, cobblestone appearance more common in the middle and lower zones, air bronchogram, which tends to be multilobar, vascular ectasia, and bronchiectasis. Atypical CT findings include mediastinal lymphadenopathy, unilateral lesions in upper zones, pleural effusion, cavitations, budding tree appearance, pneumothorax, and numerous small nodules (5).

Radiological imaging is not diagnostic for COVID19 disease. The definitive diagnosis is made with the Real-Time Polymerase Chain Reaction (RT-PCR) examination of the throat swab sample. Although this test is considered disease-specific, it has a low sensitivity in the early stages (6). During the negative phase of RT-PCR, especially in the first days of the disease, highly suspicious COVID-19 case findings were encountered quite frequently in CT. The CT findings are typical at diagnosis, and RT-PCR was found to turn positive in an average of 5.1 days (4-8 days). Among patients with negative RT-PCR tests, the probability of early detection was $93 \%$ by CT,

Copyright (C) 2021, Author(s). This is an open-access article distributed under the terms of the Creative Commons Attribution-NonCommercial 4.0 International License (http://creativecommons.org/licenses/by-nc/4.0/) which permits copy and redistribute the material just in noncommercial usages, provided the original work is properly cited 
compared to RT-PCR (7-9).

There has been no study in the literature regarding COVID-19 and the power of predicting the presence and intensity of lung involvement of the data obtained upon admission based on the chest CT severity score (CT-SS). Accordingly, this study aimed to determine the relationship of the abnormal laboratory parameters detected upon admission with CT findings and the intensity of lung involvement using CT-SS. In cases where CT cannot be performed, it will be possible to realistically estimate the predictability of abnormal laboratory values in terms of lung involvement in COVID-19 and get a chance of early intervention based on the need for hospitalization and referral to advanced centers for medical follow-up.

\section{Objectives}

This study aimed to analyze the demographic, clinical, laboratory, and radiological data of COVID-19 patients and compare the results among the determined patient groups. Furthermore, the correlation of the data obtained upon admission with the severity was examined to predict the presence of lung involvement.

\section{Methods}

\subsection{Data Sources}

The required permission was obtained from the Turkish Republic Ministry of Health, General Directorate of Health Services, and COVID-19 Scientific Research Evaluation Commission. Moreover, the study protocol was approved by our hospital Research Ethics Committee (2011-KAEK-25 2020/05-17; 27/05/2020).

This single-center study evaluated patients who were admitted to our hospital due to COVID-19 between $01 / 09 / 2020$ and $01 / 12 / 2020$ with positive PCR tests of nasal and pharyngeal swab samples and those exhibiting respiratory symptoms or fever. The patients with lung malignancy, a history of lobectomy, tuberculosis, or atelectasis were excluded from this study. A total of 242 patients who met the criteria were included in this study. Laboratory values, radiological imaging data, demographic characteristics, vital parameters upon admission, treatments administered, and clinical outcomes of the patients were recorded. According to our hospital protocol, all patients diagnosed with COVID-19 and hospitalized routinely underwent chest CT without intravenous contrast. All chest CT images were analyzed retrospectively by two experienced radiologists. The CT images were then examined upon admission, and all normal or pathological data were noted.

\subsection{Variables}

Potential variables were recorded from the time patients were first admitted to the hospital. Demographic characteristics, age, gender, smoking status, history of hypertension (HT), diabetes mellitus (DM), chronic obstructive pulmonary disease (COPD), congestive heart failure, coronary artery disease (CAD), history of extra-pulmonary malignancies, renal failure, immunosuppression, or use of immunosuppressant medications were recorded. Radiological images, such as chest radiography and thoracic CT were examined upon admission, and all normal or pathological data were noted. Clinical outcome, discharge or mortality information, duration of intensive care unit stays, and total hospital stay were also noted in this study. Vital signs including blood pressure, oxygen saturation (SaO2), and heart rate (HR) were analyzed. Laboratory data, such as complete blood count values (e.g., white blood cell count, hemoglobin-Hgb, neutrophil count, lymphocyte count, platelet countPLT), biochemical examinations (e.g., ferritin, high sensitivity troponin-I, d-dimer), sedimentation, CRP, blood urea nitrogen, creatinine, alanine aminotransferase, aspartate aminotransferase, and LDH were evaluated. Neutrophil to lymphocyte ratio (NLR) and platelet to lymphocyte ratio (PLR) were also calculated. The systemic immune inflammation index (SII) was obtained by multiplying the NLR value with the platelet count.

\subsection{Chest Computed Tomography Severity Score Assessment}

Chest CT-Severity Score (CT-SS) developed by Ran Yang et al. was used for the evaluation of chest CT images (10). The CT-SS is an adaptation of a previously used method to define ground-glass opacity, interstitial opacity, and air entrapment in post-SARS patients, all of which are associated with clinical and laboratory parameters. In CT-SS, the lung parenchyma is divided into 20 anatomical regions. In addition to 18 segments, the left upper lobe, apicoposterior segment divided into apical and posterior, and the left lower lobe anteromedial basal segment divided into anterior and medial-basal segments, add up to 20 segments in total. The CT-SS is defined by summing individual scores from 20 lung regions. Scoring depends on the intensity of pathological findings in the parenchyma for each region.

Zero, one, and two indicate no involvement, less than $50 \%$ involvement, and $50 \%$ or more involvement, respectively. The patient finally received a score between $0-40$ points according to CT findings. The patients in this study were divided into group $1(\mathrm{n}=42)$ with no involvement on thorax CT images, group $2(\mathrm{n}=123)$ with mild involvement (CTSS: $1-10)$, and group $3(\mathrm{n}=77)$ with moderate-severe involvement (CT-SS: 10 and above).

\subsection{Chest Computed Tomography Scan}

Chest CT images were acquired using a 128- 
detector CT scanner (Siemens SOMATOM®). All patients were imaged in the supine position with a single breath-hold. The scanned area extended from the apex of the lung to the costo-phrenic angle. The CT scan parameters were X-ray tube $120 \mathrm{kVp}, 350$ mAs, and slice thickness: $5 \mathrm{~mm}$.

\subsection{Statistical methods}

The obtained data were analyzed in SPSS software (IBM Statistical Package for the Social Sciences Statistic Inc. version 22.0, Chicago, IL, USA). One-way analysis of variance was used to compare three groups of ordinal variables. In our study, categorical variables were presented as frequency and percentage, while the Chi-square test was used for data analysis. Nominal variables with normal distribution were presented as mean \pm SD and compared with Student t-test, whereas non-normally distributed data were indicated as mean and interquartile range and compared with MannWhitney $U$ test (To evaluate patients as those with and without lung involvement.). The correlation between inflammatory parameters and CT involvement score was also assessed employing Pearson correlation analysis, and the predictive values of inflammatory parameters in terms of showing CT involvement were evaluated using the ROC curve analysis.

\section{Results}

The demographic characteristics of the patients $(n=242)$ included in the study and the blood parameters upon admission are presented in Table 1.

\begin{tabular}{|c|c|c|c|c|c|c|}
\hline & $\begin{array}{c}\text { Group } 1 \\
\mathrm{~N}=42 \\
\text { (none) }\end{array}$ & $\begin{array}{c}\text { Group } 2 \\
\mathrm{~N}=123 \\
\text { (mild) }\end{array}$ & $\begin{array}{c}\text { Group } 3 \\
\mathrm{~N}=77 \\
\text { (moderate-severe) }\end{array}$ & P-value* & P-value ${ }^{\dagger}$ & P-value $\neq$ \\
\hline Age & $38 \pm 10.6$ & $56.3 \pm 16$ & $61 \pm 15.6$ & $<0.001$ & $<0.001$ & 0.037 \\
\hline Male gender, n (\%) & $15(35.7 \%)$ & $52(42.3 \%)$ & $31(40.3 \%)$ & 0.755 & 0.564 & 0.829 \\
\hline Diabetes Mellitus, n (\%) & $2(4.7 \%)$ & $28(22.7 \%)$ & $27(35 \%)$ & $<0.001$ & $<0.001$ & 0.053 \\
\hline Hypertension, n (\%) & $2(4.7 \%)$ & $40(32.5 \%)$ & $32(41.5 \%)$ & $<0.001$ & $<0.001$ & 0.169 \\
\hline Coronary artery disease, $\mathrm{n}(\%)$ & $3(7.1 \%)$ & $11(8.9 \%)$ & $9(11.6 \%)$ & 0.812 & 0.196 & 0.076 \\
\hline Smoking, n (\%) & $10(23.8 \%)$ & $33(26.8 \%)$ & $22(28.6 \%)$ & 0.796 & 0.294 & 0.745 \\
\hline COPD, n (\%) & $2(4.7 \%)$ & $8(6.5 \%)$ & $5(6.4 \%)$ & 0.898 & 0.197 & 0.681 \\
\hline $\mathrm{SaO}_{2}(\%)$ & $98(94-100)$ & $97(72-100)$ & $97(78-100)$ & $<0.001$ & 0.004 & 0.077 \\
\hline Heart rate (min) & $82(64-96)$ & $84(56-144)$ & $98(56-154)$ & $<0.001$ & $<0.001$ & 0.023 \\
\hline WBC $\left(10^{3} / \mathrm{mm}^{3}\right)$ & $6,3(2,8-19,8)$ & $6(0,7-19,5)$ & $5,8(0,7-21,6)$ & 0.495 & 0.578 & 0.751 \\
\hline Hemoglobin (g/dL) & $13,2(7,9-17)$ & $13,1(5,7-16,5)$ & $12,3(1,1-16)$ & 0.395 & 0.161 & 0.063 \\
\hline Platelet $\left(10^{3} / \mathrm{mm}^{3}\right)$ & $219(47-409)$ & $219(59-507)$ & $211(20-574)$ & 0.350 & 0.576 & 0.939 \\
\hline Neutrophil $\left(10^{3} / \mathrm{ml}\right)$ & $3,4(1,1-11)$ & $3,7(0,4-18)$ & $4,1(0,4-19,1)$ & 0.165 & 0.140 & 0.083 \\
\hline Lymphocyte $\left(10^{3} / \mathrm{ml}\right)$ & $2,2(0,4-7,2)$ & $1,4(0,1-3,7)$ & $1,2(0,1-4,5)$ & $<0.001$ & $<0.001$ & 0.003 \\
\hline Neutrophil lymphocyte ratio & $1,5(0,6-11)$ & $2,4(0,3-21,9)$ & $4,7(0,7-13,9)$ & $<0.001$ & $<0.001$ & 0.001 \\
\hline Platelet lymphocyte ratio & $104,2(15,9-742,8)$ & $138,7(46,4-568)$ & $188(10,7-675,5)$ & $<0.001$ & $<0.001$ & 0.002 \\
\hline $\begin{array}{l}\text { Systemic immune inflamation } \\
\text { index }\end{array}$ & $311,7(132,6-2084,6)$ & $516.2(80-7463)$ & $1302.7(26-7454,7)$ & $<0.001$ & $<0.001$ & $<0.001$ \\
\hline Ferritin (mg/L) & $68,5(5,5-1038)$ & $188,1(6-2903)$ & $308,9(12-2903)$ & $<0.001$ & $<0.001$ & $<0.001$ \\
\hline Troponin (mg/L) & $3(0-101,6)$ & $4,5(0-653,2)$ & $5,8(0-653,2)$ & 0.392 & $<0.001$ & 0.066 \\
\hline D-dimer (ng/mL) & $0,4(0,1-38)$ & $0,6(0,1-125)$ & $0,8(0,1-16,8)$ & 0.678 & 0.001 & 0.027 \\
\hline Fibrinogen (mg/dL) & $402(122-900)$ & $456(122-995)$ & $524(122-995)$ & 0.082 & 0.032 & 0.169 \\
\hline Sedimentation $(\mathrm{mm} / \mathrm{h})$ & $20(1-70)$ & $33(1-102)$ & $44(5-116)$ & $<0.001$ & $<0.001$ & 0.030 \\
\hline C-reactive protein $(\mathrm{mg} / \mathrm{L})$ & $3,3(3-121)$ & $17,9(3-244)$ & $64,9(3,1-319)$ & $<0.001$ & $<0.001$ & $<0.001$ \\
\hline Urea (mg/dL) & $11,3(6,6-38,5)$ & $12,9(5,2-40)$ & $13,3(4,2-44,4)$ & 0.321 & 0.198 & 0.107 \\
\hline Creatinine (mg/dL) & $0,8(0,4-1,7)$ & $0,7(0,4-1,6)$ & $0,8(0,4-2,1)$ & 0.103 & 0.067 & 0.279 \\
\hline Aspartate aminotransferase (U/L) & $29(10-214)$ & $24(10-205)$ & $34(6-191)$ & 0.194 & 0.108 & 0.077 \\
\hline Alanine aminotransferase (U/L) & $18(6-206)$ & $19(5-293)$ & $25(7-261)$ & 0.214 & 0.126 & 0.109 \\
\hline Lactate dehydrogenase (U/L) & $170(112-464)$ & $245(120-617)$ & $308(124-742)$ & $<0.001$ & $<0.001$ & $<0.001$ \\
\hline
\end{tabular}

COPD: Chronic obstructive pulmonary disease, WBC: White blood cells

${ }^{*}$ Comparison of the three groups

${ }^{+}$Comparison of patients with or without lung involvement

^Comparison of patients with mild or moderate-severe lung involvement

The mean ages of groups $1(n=42), 2(n=123)$, and $3(n=77)$ were $38 \pm 10.6,56.3 \pm 16$, and $61 \pm 15.6$ years, respectively $(\mathrm{P}<0.001)$. The three groups were similar in terms of gender, smoking, presence of CAD, COPD, kidney and liver functions; however, they significantly differed in terms of HT, DM, HR, $\mathrm{SaO2}$, lymphocyte count, PLR, SII, ferritin, troponin, erythrocyte sedimentation rate (ESR), and CRP values $(\mathrm{P}<0.001)$. Age, DM and HT rates, heart rate, NLR, PLR, SII, ferritin, troponin, ESR, and CRP values were significantly higher in groups 2 and 3 $(\mathrm{P}<0.001)$ (Table 1). The patients were evaluated as those with mild ( $\mathrm{n}=123$, group 2$)$ and moderatesevere $(n=77$, group 3) lung involvement. Age, heart rate, lymphocyte count, NLR, PLR, SII, ferritin, ESR, and CRP values were significantly higher in patients with lung involvement $(\mathrm{P}=0.037$, $\mathrm{P}=0.023, \quad \mathrm{P}=0.003, \mathrm{P}=0.001, \mathrm{P}=0.002, \mathrm{P}<0.001$, $\mathrm{P}<0.001, \mathrm{P}=0.030$, and $\mathrm{P}<0.001$, respectively) (Table 1). The ROC analysis was performed for 
inflammatory parameters in terms of showing lung involvement. The cut-off values for CRP, ferritin, SII, NLR, PLR, and ESR were obtained at 8.91 (75.3\% sensitivity, 75\% specificity), 132.6 (72.2\% sensitivity, $\quad 72.7 \% \quad$ specificity), $436 \quad(70.7 \%$ sensitivity, $\quad 72.7 \%$ specificity), $\quad 2.13 \quad(69.2 \%$ sensitivity, $\quad 68.2 \%$ specificity), $127.9 \quad(67.7 \%$ sensitivity, $68.2 \%$ specificity), and 23.5 (64.6\% sensitivity, 65.9\% specificity), respectively (Table 2) (Figure 1).

Correlation analysis was performed between CTSS and inflammatory parameters (Figure 2).

\begin{tabular}{|c|c|c|c|c|c|c|}
\hline Variables & Cut-off & AUC & 95\% CI & Sensitivity & Specificity & P-value \\
\hline C-reactive protein & 8.91 & 0.825 & $0.764-0.885$ & 75.3 & 75 & $<0.001$ \\
\hline Ferritin & 132.6 & 0.787 & $0.710-0.863$ & 72.2 & 72.7 & $<0.001$ \\
\hline Systemic immune inflammation index & 436 & 0.746 & $0.676-0.817$ & 70.7 & 72.7 & $<0.001$ \\
\hline Neutrophil lymphocyteratio & 2.13 & 0.743 & $0.669-0.817$ & 69.2 & 68.2 & $<0.001$ \\
\hline Platelet lymphocyteratio & 127.9 & 0.729 & $0.646-0.812$ & 67.7 & 68.2 & $<0.001$ \\
\hline Erythrocyte sedimentation rate & 23.5 & 0.705 & $0.631-0.780$ & 64.6 & 65.9 & $<0.001$ \\
\hline
\end{tabular}

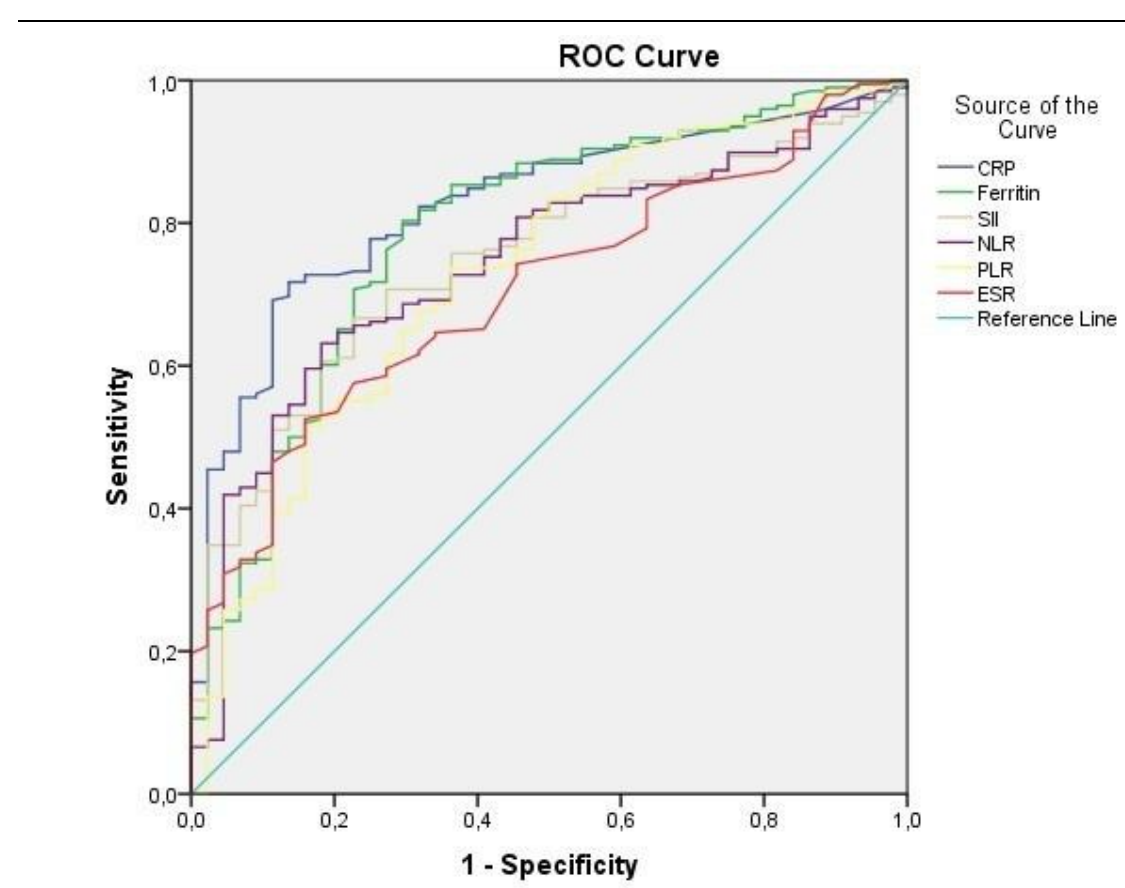

Diagonal segments are produced by ties.

Figure 1. Receiver operating characteristic curve analysis figure for blood parameters
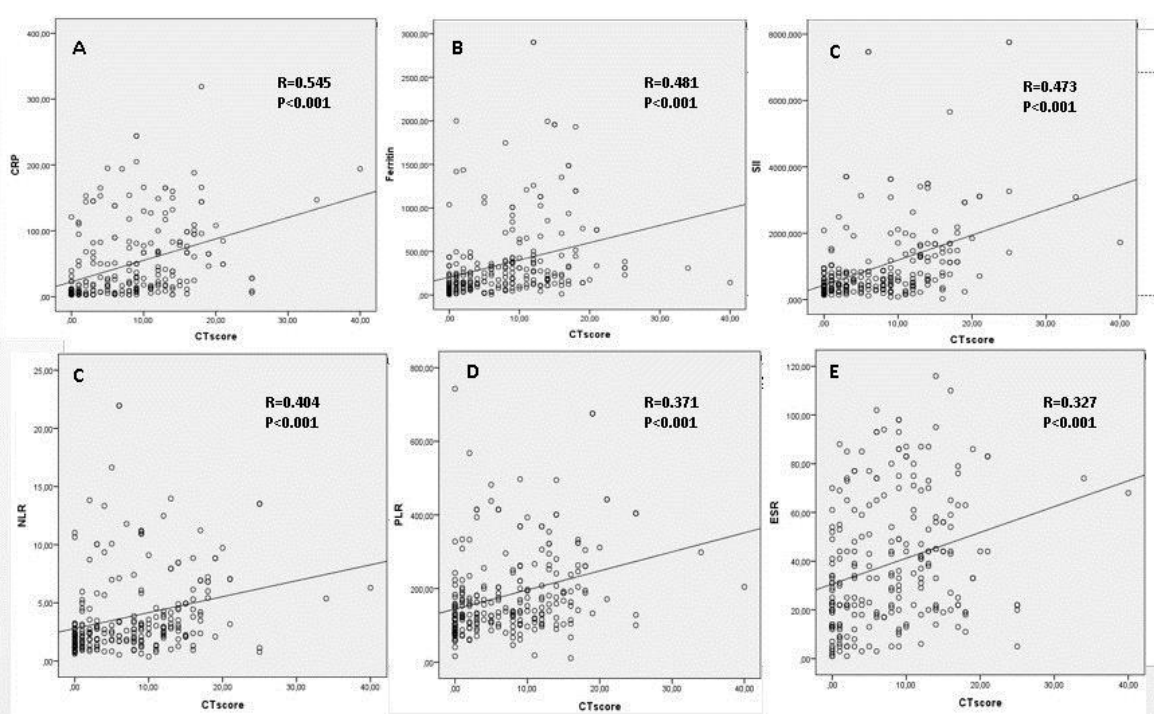

Figure 2. Correlation analysis between computed tomography scores and biomarkers 
Accordingly, CT-SS and CRP ( $\mathrm{R}=0.545, \mathrm{P}<0.001)$, ferritin $(\mathrm{R}=0.481, \quad \mathrm{P}<0.001)$, and SII $(\mathrm{R}=0.473$, $\mathrm{P}<0.001)$ were moderately and positively correlated, while NLR $(\mathrm{R}=0.404, \mathrm{P}<0.001)$, PLR $(\mathrm{R}=0.371$, $\mathrm{P}<0.001)$, and $\operatorname{ESR}(\mathrm{R}=0.327, \mathrm{P}<0.001)$ were mildly and positively correlated.

\section{Discussion}

As the first year of the COVID-19 pandemic is left behind, it has become clear that the world, especially the healthcare systems, was not prepared for such a major crisis. The current data (11) shared by the Centers for Disease Control and Prevention and some additional publications made it clear that races, ethnic identities, and geographical regions differed in terms of access to healthcare, and therefore, mortality rates (12-14). As of 01 April 2021, COVID19 infection resulted in the death of almost three million individuals, and infection of more than 125 million and increasing number of cases. These numbers are also an indicator of the workload on health institutions. There is a great burden on clinicians, especially physicians who meet patients first, due to explosively increased visits to healthcare institutions. It has become very important to identify the patients, interpret the clinical findings and laboratory data correctly as of the first visit, and determine the effective diagnosis-follow-uptreatment strategy. Early detection of COVID-19associated pneumonia, which is primarily associated with mortality during the disease process, should be the main goal.

This study aimed to develop an early diagnosis system that allows the clinician to predict lung involvement and its severity, as well as the likelihood of pulmonary complications based on easily obtained laboratory data. The COVID-19 symptomatology ranges within a wide spectrum from asymptomatic carriage to pneumonia with extensive lung involvement, multiple organ failure, and sepsis. Most patients overcome the disease with mild or moderate severity. The severe disease group was found to have comorbidities, such as HT, DM, CAD, immune deficiency, or malignancy. In addition, diseasespecific elevations in laboratory parameters, such as d-dimer, troponin-I, LDH, ferritin, and CRP have been reported (15-18).

Comprehensive analyses state that the incidence of severe disease and the need for hospitalization are approximately between $12 \%$ and $16 \%(19,20)$, and those of severe pneumonia and critical disease range between $14-35 \%$ and $5-28 \%$, respectively (4, 15 ,). On the other hand, in patients with suspected COVID-19 lesions on CT, the RT-PCR test, which provides diagnostic precision for COVID-19, turns positive in an average of 5.1-6.2 days (7-9). All these data show that early diagnosis can be achieved thanks to possible correlations between clinical complaints, laboratory data, and CT findings before RT-PCR positivity is found. The CT-SS shows the severity of lung involvement. The same scoring system was used effectively in the SARS epidemic in previous years. In the SARS epidemic, pathological findings in the lung parenchyma were correlated with laboratory and clinical parameters (21). In studies conducted on patients with COVID-19 pneumonia which examined the relationship between CT-SS and clinical severity, CT-SS was proven as an effective scoring system for showing the severity of the disease in COVID-19-associated pneumonia $(10,22-25)$.

Our three patient groups significantly differed in terms of $\mathrm{HT}$ and DM rates, HR, SaO2, lymphocyte count, PLR, SII, ferritin, troponin-I, ESR, and CRP values $(\mathrm{P}<0.001$ for all). Among 200 patients with pulmonary involvement, age, heart rate, as well as DM and HT rates were significantly higher, compared to those without $(\mathrm{P}<0.001)$.

Similarly, in patients with lung involvement, NLR, PLR, SII, ferritin, troponin, ESR, and CRP values were significantly higher $(\mathrm{P}<0.001)$. Similar to our findings, several studies have shown that the development of serious disease is more pronounced in the presence of comorbid diseases, such as HT, DM, and malignancy $(15,26,27)$. In a study involving 32,583 patients, it is mentioned that the risk of contracting the disease and developing serious disease increases in the presence of at least one of the comorbidities, such as obesity, DM, and HT (28). In addition to these, there are publications stating that some abnormalities detected in blood parameters and biochemical variables also cause an increase in the incidence of serious diseases and a greater predisposition to the development of mortality (2932 ). In our study, similar to the literature, significant increases were observed in the presence of comorbid diseases and some laboratory parameters in terms of the presence and prevalence of lung involvement. Additionally, in our study, ferritin, SII, and CRP levels were found to be significantly higher among the patients who were divided into two groups with CTSS in terms of parenchymal involvement severity (Table 1). In this way, significant results were obtained in terms of both the presence of involvement and the determination of the severity of the involvement.

The ROC analysis performed with inflammatory parameters revealed that SII, NLR, PLR, and ESR, especially CRP and ferritin, were the strong predictors of lung involvement with high specificity and sensitivity (CRP>8.91; AUC: 0.825, 75.3\% sensitivity and $75 \%$ specificity, ferritin $>132.6$; AUC: $0.787,72.2 \%$ sensitivity and $72.7 \%$ specificity, $\mathrm{P}<0.001)$. According to the correlation analysis performed between CT-SS and inflammatory data, the CT-SS score was moderately and positively correlated with $C R P(R=0.545, \quad P<0.001)$, ferritin $(R=0.481$, 
$\mathrm{P}<0.001)$, and SII $(\mathrm{R}=0.473, \mathrm{P}<0.001)$; however, it was mildly and positively correlated with NLR $(\mathrm{R}=0.404, \mathrm{P}<0.001)$, PLR $(\mathrm{R}=0.371, \mathrm{P}<0.001)$, and $\operatorname{ESR}(\mathrm{R}=0.327, \mathrm{P}<0.001)$.

These significant data, especially CRP, ferritin, and SII were highly sensitive and specific upon the first admission in terms of predicting lung parenchymal involvement due to COVID-19 without CT scans. Thanks to this predictive power, it is believed that negative conditions, such as living in rural areas, having low socio-economic opportunities, not having advanced examination options, such as CT in the country or region of residence, or not being able to benefit from health services can be effectively prevented. As a secondary gain, it is our opinion that unnecessary CT scans obtained to investigate lung involvement can also be avoided. Blood data obtained from patients upon admission may alert the clinician primarily for the presence of lung involvement. This way, early treatment opportunities will be offered to patients with pneumonic involvement during the average five-day window of RT-PCR negativity. With significant elevations in CRP, ferritin, and SII, the presence of lung involvement can be predicted and the selection of patients in need of hospitalization and referral to advanced centers for examination and treatment will get much easier.

This study consists of patients who were hospitalized with the diagnosis of COVID-19 and received treatment. Therefore, the number of patients is low. The other significant limitations of the present study are its retrospective and single-center study design.

\section{Conclusion}

Timely determination of the disease course and taking measures accordingly are of great importance for healthcare systems battling with the pandemic. Early detection of morbidity development, the need for hospitalization and pneumonia, as well as developing a diagnostic and treatment strategy will ensure the equal distribution of the increased burden on the health systems. This study revealed that abnormal laboratory findings detected in patients upon admission are effective in predicting lung involvement with high specificity and sensitivity without the use of a thoracic CT scan. This information will greatly help the clinician who manages the COVID-19 patient first in terms of prediction of lung involvement. High values of CRP, ferritin, and SII lead to the identification of patients with parenchymal involvement, and the intensity of parenchymal involvement in these patients will be noticed more clearly.

It is believed that by using this ability, the need for CT will decrease, the economic burden on health systems will become more balanced, the management of the disease will be more selective, and the fight against the pandemic will be carried out more effectively. Further multicenter prospective studies with large patient series are required for clearer results.

\section{Acknowledgments}

The authors would like to thank all the medical staff for their efforts in combating the outbreak of COVID-19.

\section{Footnotes}

Authors' Contribution: YA: Substantial contributions to the conception or design of the work; or the acquisition, analysis, or interpretation of data for the work; drafting the work or revising it critically for important intellectual content; final approval of the version to be published; AKA: Substantial contributions to the conception or design of the work; or the acquisition, analysis, or interpretation of data for the work; drafting the work or revising it critically for important intellectual content; final approval of the version to be published; ME: Drafting the work or revising it critically for important intellectual content; final approval of the version to be published; NKK: Drafting the work or revising it critically for important intellectual content; final approval of the version to be published; TS: Drafting the work or revising it critically for important intellectual content; final approval of the version to be published; SAS: Drafting the work or revising it critically for important intellectual content; final approval of the version to be published; CE: Drafting the work or revising it critically for important intellectual content; final approval of the version to be published; UA: Drafting the work or revising it critically for important intellectual content; final approval of the version to be published; FA: Interpretation of data for the work; drafting the work or revising it critically for important intellectual content; final approval of the version to be published; TT: Drafting the work or revising it critically for important intellectual content; final approval of the version to be published

Conflicts of Interest: There is no conflict of interest regarding the publication of the study.

Ethical Approval: Ethical issues including plagiarism, data fabrication, and double publication have been completely observed by the authors. The study followed the tenets of the Declaration of Helsinki and was approved by Bursa Yuksek Ihtisas Training and Research Hospital Clinical Research Ethics Committee dated 27/05/2020 and protocol numbered 2011-KAEK-25 2020 / 05-17.

\section{Funding/ Support: None}

Financial Disclosure: None 
Informed Consent: Written informed consent was taken from all participants before any intervention.

\section{References}

1. Zhu N, Zhang D, Wang W, Li X, Yang B, Song J, et al. A novel coronavirus from patients with pneumonia in China, 2019. $N$ Engl $J$ Med. 2020;382(8):727-33. doi: 10.1056/NEJMoa2001017. [PubMed: 31978945].

2. Riahi T, Shokri S, Faiz SHR, Hemati K, Mousavie SH, Baghestani $A$, et al. Clinical and laboratory predictors of mortality in hospitalized COVID-19 patients. Iran Red Crescent Med J. 2021;23(5):e531. doi: 10.32592/ircmj.2021.23.5.531.

3. Wang C, Horby, PW, Hayden FG, Gao GF. A novel coronavirus outbreak of global health concern. Lancet. 2020;395(10223): 470-3. doi: 10.1016/S0140-6736(20)30185-9. [PubMed: 31986257].

4. Wu Z, McGoogan JM. Characteristics of and important lessons from the coronavirus disease 2019 (COVID-19) outbreak in china: summary of a report of 72314 cases from the Chinese center for disease control and prevention. JAMA. 2020;323(13):1239-42. doi: 10.1001/jama.2020.2648. [PubMed: 32091533].

5. Ye Z, Zhang Y, Wang Y, Huang Z, Song B. Chest CT manifestations of new coronavirus disease 2019 (COVID-19): a pictorial review. Eur Radiol. 2020;30(8):4381-9. doi: 10.1007/s00330-020-06801-0. [PubMed: 32193638].

6. Li X, Geng M, Peng Y, Meng L, Lu S. Molecular immune pathogenesis and diagnosis of COVID-19. J Pharm Anal. 2020;10(2):102-8. doi: 10.1016/j.jpha.2020.03.001. [PubMed: 32282863].

7. Ai T, Yang Z, Hou H, Zhan C, Chen C, Lv W, et al. Correlation of chest CT and RT-PCR testing for coronavirus disease 2019 (COVID-19) in China: a report of 1014 cases. Radiology. 2020;296(2):E32-40. doi: 10.1148/radiol.2020200642. [PubMed: 32101510].

8. Xie X, Zhong Z, Zhao W. Chest CT for typical coronavirus disease 2019 (COVID-19) pneumonia: relationship to negative RT-PCR testing. Radiology. 2020;296(2):E41-5. doi: 10.1148/radiol.2020200642. [PubMed: 32049601].

9. Fang Y, Zhang H, Xie J, Lin M, Ying L, Pang P, Ji W. Sensitivity of chest CT for COVID-19: comparison to RT-PCR. Radiology. 2020;296(2):E115-7. doi: 10.1148/radiol.2020200432. [PubMed: 32073353].

10. Yang R, Li X, Liu H, Zhen Y, Zhang X, Xiong Q, et al. Chest CT severity score: an imaging tool for assessing severe COVID-19. Radiol Cardiothorac Imaging. 2020;2(2):e200047. doi: 10.1148/ryct.2020200047. [PubMed: 33778560].

11. Kim L, Whitaker M, O'Halloran A, Kambhampati A, Chai SJ, Reingold A, et al. COVID-NET surveillance team. hospitalization rates and characteristics of children aged $<18$ years hospitalized with laboratory-confirmed COVID-19 - COVID-NET, 14 States, March 1-July 25, 2020. MMWR Morb Mortal Wkly Rep. 2020;69(32):1081-8. doi: 10.15585/mmwr.mm6932e3. [PubMed: 32790664].

12. Khunti K, Singh AK, Pareek M, Hanif W. Is ethnicity linked to incidence or outcomes of covid-19? BMJ. 2020;369:m1548. doi: 10.1136/bmj.m1548. [PubMed: 32312785].

13. Karaca-Mandic P, Georgiou A, Sen S. Assessment of COVID-19 hospitalizations by race/ethnicity in 12 states. JAMA Intern Med. 2021;181(1):131-4. doi: 10.1001/jamainternmed.2020.3857. [PubMed: 32804192].

14. Bhala N, Curry G, Martineau AR, Agyemang C, gyemang C, Bhopal R. Sharpening the global focus on ethnicity and race in the time of COVID-19. Lancet. 2020;395(10238): 1673-6. doi: 10.1016/S0140-6736(20)31102-8. [PubMed: 32401716].

15. Zhou F, Yu T, Du R, Fan G, Liu Y, Liu Z, et al. Clinical course and risk factors for mortality of adult inpatients with COVID-19 in Wuhan, China: a retrospective cohort study. Lancet. 2020;395(10229):1054-62. doi: 10.1016/S01406736(20)30566-3. [PubMed: 32171076].

16. Liu H, Chen S, Liu M, Nie H, Lu H. Comorbid chronic diseases are strongly correlated with disease severity among COVID-19 patients: a systematic review and meta-analysis. Aging Dis. 2020;11(3):668-78. doi: 10.14336/AD.2020.0502. [PubMed: 32489711].

17. Yang J, Zheng Y, Gou X, Pu K, Chen Z, Guo Q, et al. Prevalence of comorbidities and its effects in patients infected with SARSCoV-2: a systematic review and meta-analysis. Int J Infect Dis. 2020;94:91-5. doi: 10.1016/j.ijid.2020.03.017. [PubMed: 32173574]

18. Zhang JJ, Cao YY, Tan G, Dong X, Wang BC, Lin J, et al. Clinical, radiological, and laboratory characteristics and risk factors for severity and mortality of 289 hospitalized COVID-19 patients. Allergy. 2021;76(2):533-50. doi: 10.1111/all.14496. [PubMed: 32662525].

19. Guan WJ, Ni ZY, Hu Y, Liang WH, Ou CQ, He JX, et al. Clinical characteristics of coronavirus disease 2019 in China. $N$ Engl J Med. 2020;382(18):1708-20. doi: 10.1056/NEJMoa2002032.

20. Garg S, Kim L, Whitaker M, O'Halloran A, Cummings C, Holstein R, et al. Hospitalization rates and characteristics of patients hospitalized with laboratory-confirmed coronavirus disease 2019 - COVID-NET, 14 States, March 130, 2020.MMWR Morb Mortal Wkly Rep. 2020;69(15):45864. doi: 10.15585/mmwr.mm6915e3. [PubMed: 32298251].

21. Chang YC, Yu CJ, Chang SC, Galvin JR, Liu HM, Hsiao CH, et al. Pulmonary sequelae in convalescent patients after severe acute respiratory syndrome: evaluation with thin-section CT. Radiology. 2005;236(3):1067-75. doi: 10.1148/radiol.2363040958. [PubMed: 16055695].

22. Francone M, Iafrate F, Masci GM, Coco S, Cilia F, Manganaro L, et al. Chest CT score in COVID-19 patients: correlation with disease severity and short-term prognosis. Eur Radiol. 2020;30(12):6808-17. doi: 10.1007/s00330-020-07033-y. [PubMed: 32623505].

23. Zhang J, Meng G, Li W, Shi B, Dong H, Su Z, et al. Relationship of chest CT score with clinical characteristics of 108 patients hospitalized with COVID-19 in Wuhan, China. Respir Res. 2020;21(1):180. doi: 10.1186/s12931-020-01440-x. [PubMed: 32664991].

24. Inui S, Fujikawa A, Jitsu M, Kunishima N, Watanabe S, Suzuki Y, et al. Erratum: chest CT findings in cases from the cruise ship "diamond princess" with coronavirus disease 2019 (COVID19). Radiol Cardiothorac Imaging. 2020;2(2):e204002. doi: 10.1148/ryct.2020200110. [PubMed: 33779623].

25. Lessmann N, Sánchez CI, Beenen L, Boulogne LH, Brink M, Calli E, et al. Automated assessment of COVID-19 reporting and data system and chest CT severity scores in patients suspected of having COVID-19 using artificial intelligence. Radiology. 2021;298(1):E18-28. doi: 10.1148/radiol.2020202439. [PubMed: 32729810].

26. Huang C, Wang Y, Li X, Ren L, Zhao J, Hu Y, et al. Clinical features of patients infected with 2019 novel coronavirus in Wuhan, China. Lancet. 2020;395(10223):497-506. doi: 10.1016/S0140-6736(20)30183-5. [PubMed: 31986264].

27. Huang S, Wang J, Liu F, Liu J, Cao G, Yang C, et al. COVID-19 patients with hypertension have more severe disease: a multicenter retrospective observational study. Hypertens Res. 2020;43(8):824-31. doi: 10.1038/s41440-020-0485-2. [PubMed: 32483311].

28. Hernández-Garduño E. Obesity is the comorbidity more strongly associated for Covid-19 in Mexico. A case-control study. Obes Res Clin Pract. 2020;14(4):375-9. doi 10.1016/j.orcp.2020.06.001. [PubMed: 32536475].

29. As AK, Erdolu B, Duman B, Yazgan E, Eris C, Aydin U, et al. Can a modified-simplified pulmonary embolism severity index (msPESI) be used to predict the need for intensive care in hospitalized COVID-19 patients? I Thromb Thrombolysis. 2021;12:1-7. doi: 10.1007/s11239-021-02405-7. [PubMed: 33710508].

30. Jose RJ, Manuel A. COVID-19 cytokine storm: the interplay between inflammation and coagulation. Lancet Respir Med. 2020;8(6):e46-7. doi: 10.1016/S2213-2600(20)30216-2. [PubMed: 32353251].

31. Shang W, Dong J, Ren Y, Tian M, Li W, Hu J, et al. The value of clinical parameters in predicting the severity of COVID-19. J 
Med Virol. 2020;92(10):2188-92. doi: 10.1002/jmv.26031. [PubMed: 32436996].

32. Cummings MJ, Baldwin MR, Abrams D, Jacobson SD, Meyer BJ, Balough EM, et al. Epidemiology, clinical course, and outcomes of critically ill adults with COVID-19 in New York City: a prospective cohort study. Lancet. 2020;395 (10239):1763-70. doi: 10.1016/S0140-6736(20)31189-2. [PubMed: 32442528] 